\title{
On the Performance Bounds of LTE- A System under Varied UE Distributions
}

\author{
Navneet Kaur $^{1}$, Jyoteesh Malhotra ${ }^{2}$ \\ Dept. of Electronics and Communications Engineering; GNDU Regional Campus; Jalandhar; India
}

\begin{abstract}
The Universal Mobile Telecommunications System (UMTS) adapted the LTE. It permits consumers of mobile to attain Internet over several devices. The main aim of the LTE is to provide a steep data transmission rate and many more audiovisual services. Scheduling is that kind of procedure in which distribution of resources to the consumers takes place to get improved amount. The scheduler allocates the common resources i.e. frequency as well as time amongst consumer's stations. In this paper, we have taken the Round Robin scheduling algorithm, the simulation and the examination of the scheduling procedures stayed complete by imitations accomplished on a MATLAB-build downlink link level simulator by the University of Vienna. We accomplish our observation of results that clearly shows the variation in throughput, speed, radii as well as fairness, numerous results are exposed which displays examination and achieves data that upsurges the wireless interacting.
\end{abstract}

Keywords: RB (Resource Blocks), Round Robin, Throughput, Noise density.

\section{Introduction}

In the system of the LTE- downlink, scheduling must be achieved through 1 TTI, it contains of binary time periods, or resource-block-pair foundation (RB, one sub-frame which is of $0.5 \mathrm{~ms}$ above $180 \mathrm{kHz}$ ). Round Robin is primarily and extensively used procedure. The process of round robin is expansively used because of its easier implementation. The RR [1] allocates equivalent slices of packet broadcast period to individual user in a round direction. The fairness performance is highly achieved by the RR. This is that type of process which uses first come first served concept. Furthermore, RR springs each UE an equivalent chance to acquire RB's, the whole throughput is abundant inferior than further schedules since the RR not ensures the condition of channel. In LTE, dissimilar UEs must have altered facilities with QoS necessities and it is very problematic to permit each SN for similar RBs for the equivalent option since it will decline the resource effectiveness [2]. The downlink physical source is characterized by a time-frequency reserve network containing of numerous Resource Blocks (RB). A resource block is distributed in some resource elements (RE). A scheduler is a significant component in the BS and it allocates the frequency and time possessions to dissimilar consumers in the cell. Consequently, a RB is the minutest component which is allocated through the scheduler. This handout describes the RR only and clearly shows the results in terms of various kinds of throughput, fairness as well. In Round Robin (RR) scheduling [3] the stations are allocated the RB's one next another deprived of CQI. Consequently, the scheduling of the stations is likewise arranged. The inspiration to effort on this scheme originates from the point that LTE is the upcoming of portable broadband. It is predictable in order that in the upcoming $80 \%$ of wholly mobile broadband consumers would be assisted by LTE [4]. Therefore, one and only of the chief aim of LTE is to deliver actual well-organized control equivalent devices to attain longer lifespan of battery and efficiently assign the Resource blocks [5]. The paper is prepared in numerous segments, first part is introductory section. Next part is the LTE radio resource scheduling algorithm which is having further two segments i.e. Scheduling and Round Robin. The third section of this handout shows the contextual explanation of various parameters. The fourth part describes the simulation methodology and simulation environment. The last part discuss about the consequences of the RR.

\section{A. Scheduling}

\section{LTE Radio Resource Scheduling Algorithm}

In OFDM scheme the wireless resource arrangement is considered. Scheduling is the procedure of assigning resources to the consumer to become improved throughput, reasonable distribution of resource although reducing packet interruption and packet damage inside QoS necessity. Moreover many forms of scheduling algorithms are available but we will consider only the RR scheduling algorithm in this handout. In Round Robin (RR) scheduling [6] the stations are allocated the RB's one next another deprived of CQI to each consumer in definite time break. The process of allocating the resource is that firstly the consumer will use the resource for the specified time break and afterwards the achievement of time then these available resources is allocated to a new consumer. The fresh consumer has positioned at the finale of coming up line. The RR is one of at ease algorithm with easy implementation, it is having virtuous fairness. We chose the Round Robin scheduling since of the good features. The Round Robin scheduling is equitable in the extended period meanwhile it similarly plans the MS.

\begin{tabular}{|c|c|}
\hline DOI: $10.9790 / 2834-1203028695$ & www.iosriournals.org \\
\hline
\end{tabular}




\section{B. Round Robin Scheduling}

In Round Robin (RR) scheduling [3] the stations are allocated the RB's one next another deprived of CQI. Consequently, the scheduling of the stations is likewise arranged shown in fig.1 [7].

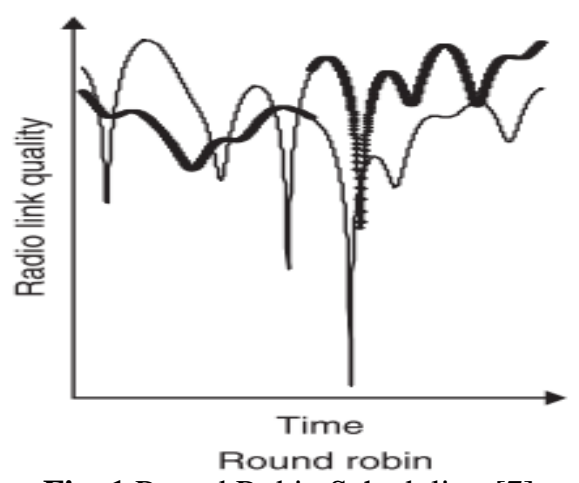

Fig. 1 Round Robin Scheduling [7]

The main benefit of Round Robin scheduling is the promise of fairness for totally consumers. Additionally Round Robin is relaxed to executed, so is the aim is typically used by numerous schemes. Subsequently Round Robin not take the channel quality data into justification, it resolve outcomes in small consumer amount. The step by step chart of the Round Robin Scheduling is as displayed under:

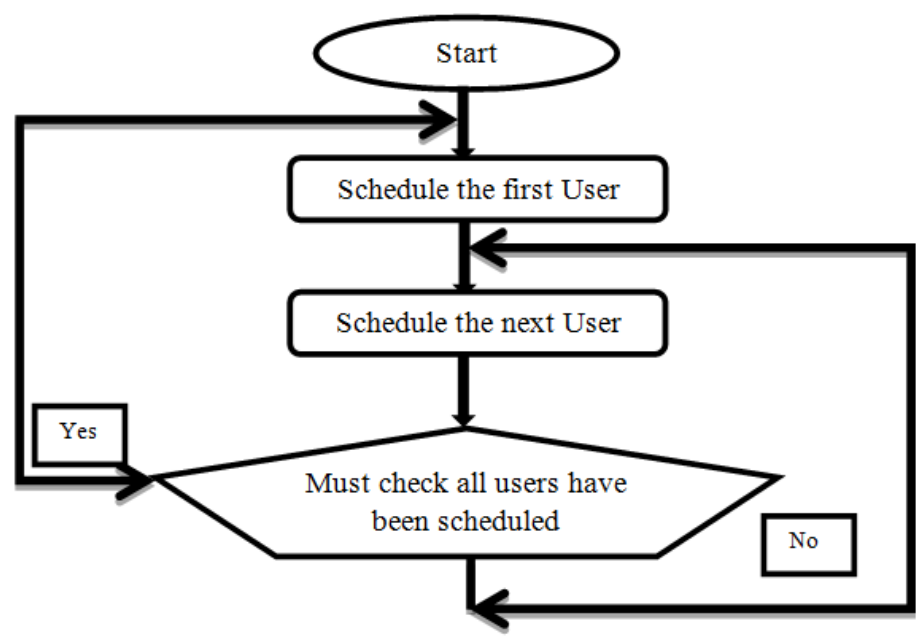

Fig. 2 Flowchart of Round Robin Scheduling

\section{Contexual Explanation}

In order to describe the performance evaluation results related to LTE wireless system the important modes and the channel behavior need to be elaborated. This section gives the brief summary of the background description in this regard as enumerated below:

\section{A. Noise Figure}

Noise figure (NF) and noise factor $(F)$ are procedures of deprivation of SNR, affected due to modules in a radio-frequency (RF) pointer series. It is a quantity through where the presentation of an amplifier or a radio receiver can be quantified, by means of inferior standards representative improved enactment. The noise factor is well-defined as the proportion of the device's output noise power to the serving thereof attributable to input thermal noise at typical noise temperature $T_{0}$ i.e. typically $290 \mathrm{~K}$.

The noise figure is just the noise factor articulated in decibels $(\mathrm{dB})$. It is expressed as:

B.

$$
\mathrm{NF}=\frac{\mathrm{SNR}_{\text {in }}}{\mathrm{SNR}_{\text {out }}}
$$

where $\mathrm{SNR}_{\text {in }}$ and $\mathrm{SNR}_{\text {out }}$ are the input and output signal-to-noise ratios correspondingly. 


\section{B. Thermal Noise Density}

In communications, Noise density $\left(N_{0}\right)$ is the power spectral density of noise or we can say that the noise power each part of bandwidth. It has measurement of power above frequency and SI unit stays watts per hertz i.e. corresponding to watt-seconds. For thermal noise, spectral density is: $N_{0}=k T$, where $k$ is Boltzmann's constant which is represented in joules per kelvin, and also $T$ is the noise temperature for receiver which is represented in kelvins.

\section{C. eNodeB}

The Evolved Node B is the component of E-UTRA of LTE which is the advancement of the component Node B which is present in Node B of UMTS. The eNodeB is hardware which is associated with the network of mobiles which interconnects straight without wires by the whole of mobile users i.e. UEs. The eNodeB is totally under the control of RNC (Radio Network Controller), no dispersed controller component takes place.

\section{A. Flow chart of the program}

\section{Simulation Methodology And Environment}

The discrete happening replication approved out in this handout used MATLAB utilities and scripts.

The procedure of sequence of simulation has been described below:

- Start the program and fixed global debug level to 1

- Adjust the considerations of basic configuration

- Open the LTE_load_params.m file

- Set the transmitter power to $49 \mathrm{dBm}$ and bandwidth $=1.4^{*} 10^{\wedge} 6 \mathrm{MHz}$

- Determine transmission mode values equivalent to no. of nTX and nRX

- $\quad$ Set scheduler $=$ Round Robin

- Set additional configuration parameters for the scheduler

- Set Channel model type = gamma and network geometry= Regular hexagonal grid

- Now select distance between eNodeB's and no. of eNodeB's rings

- Define type of fading= Claussen

- Set values of additional parameters related to fading

- Select UE receiver noise figure value which is in $\mathrm{dB}$.

- Choose the UE thermal noise density value for the SISO and MIMO as well

- Now, set UE per eNodeB values ie. 10,25,2

- Determine Outputs for corresponding thermal noise density

- Case 1: UE per eNodeB values $=2$, Case 2: UE per eNodeB values $=10$, UE per eNodeB values $=25$

- For scheduler, simulation decide and simulation type= tri_sector_tilted_4*4

- Simulation Starts

- Load in load_params_file

- No. of outputs shown and results save in result file

- Exit

\section{B. Simulation Environment}

The Simulation considerations as per the PHY and MAC layer conditions used in the replications have been tabularized below:

Table 1: Simulation Environment

\begin{tabular}{|c|c|c|}
\hline Sr. no. & Parameters & Values \\
\hline 1. & Bandwidth & MHz \\
\hline 2. & Transmitted power & $2.14 \mathrm{dBH}$ \\
\hline 3. & Carrier Frequency & Tri_sector_tilted_4*4 \\
\hline 4. & Simound Robin \\
\hline 5. & Scheduler & generated \\
\hline 6. & Simulation tti & CLSM \\
\hline 7. & Transmission mode & 2 \\
\hline 8. & No. of transmitters & 2 \\
\hline 9. & No. of receivers & Homogeneous \\
\hline 10. & Power allocation & Gamma \\
\hline 11. & Channel model type & 8Km/hr, 10Km/hr \\
\hline 12. & UE_Speed & Claussen \\
\hline 13. & Shadow fading type & $13,20 \mathrm{~dB}$ \\
\hline 14. & UE receiver noise figure & 2,25 \\
\hline 15. & UE per eNodeB & Regular Hexagonal grid \\
\hline 16. & Network geometry & $450 \mathrm{~m}$ \\
\hline 17. & eNodeB distance & 2 \\
\hline 18. & eNodeB rings & \\
\hline 19. & & \\
\hline
\end{tabular}




\section{Results And Discussions}

Based on the extensive simulations that have been carried out as per the details mentioned in the previous section, the following results have been obtained:

\section{A. Small Scale Fading type}

Following are the results shows regarding the variation in speed. By changing speed, it effects on UE and eNodeB's. We have taken the value of speed i.e. $10 \mathrm{Km} / \mathrm{hr}$.

\section{Speed $=10 \mathrm{Km} / \mathrm{hr}$}

When we have choose the speed $=10 \mathrm{Km} / \mathrm{hr}$ then above are the results takes place. In Fig.a shows the eNodeB 14 and sectors correspondingly are 1,2,3. The total number of eNodeB's are 20 and each having their respective sectors. Next fig.b shows about the shadow fading sites ie. from 1-19, each site is having value from -1000 to 1000. The fig. c shows the correlated shadow fading maps with normal distribution. The curve shows two colored lines, the red line shows the normal distribution and black line shows the correlated shadow fading maps.

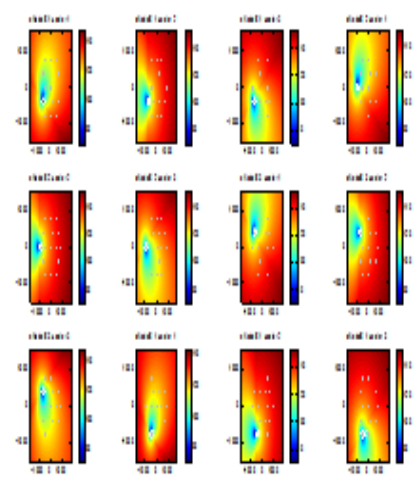

Fig.a: eNodeB 1-4
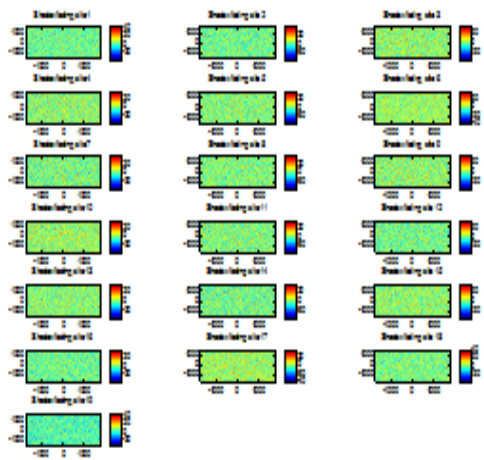

Fig.b: Shadow Fading sites

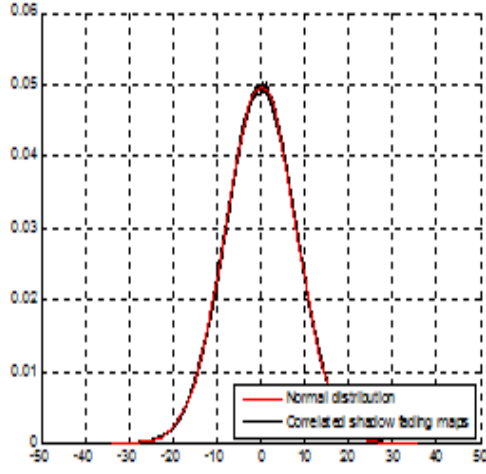

Fig.c: Shadow Fading maps

Next, the fig.d shows the flower diagrams that shows about ROI max SINR (SISO, macroscopic and shadow fading) and the no. of cells are nominated i.e. 1-19. It also shows SISO CQI mapping that displays for macroscopic and shadow fading, SINR alteration caxis limited to $15 \mathrm{~dB}$, eNodeB consignment for macroscopic and shadow fading. On the other hand, fig.e also shows overhead specified influences. The only modification in fig. $\mathrm{d}$ and fig. $\mathrm{e}$ is that in fig. $\mathrm{d}$ shadow fading exists and in the fig. e only macroscopic fading displays.
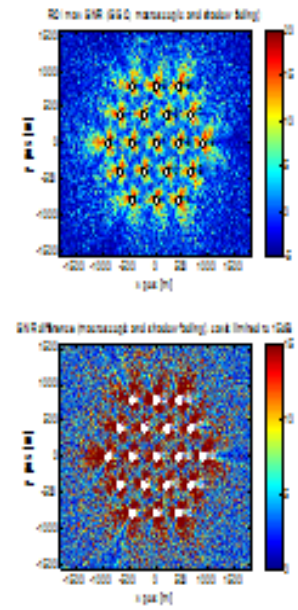

Fig.d: Macroscopic and Shadow Fading
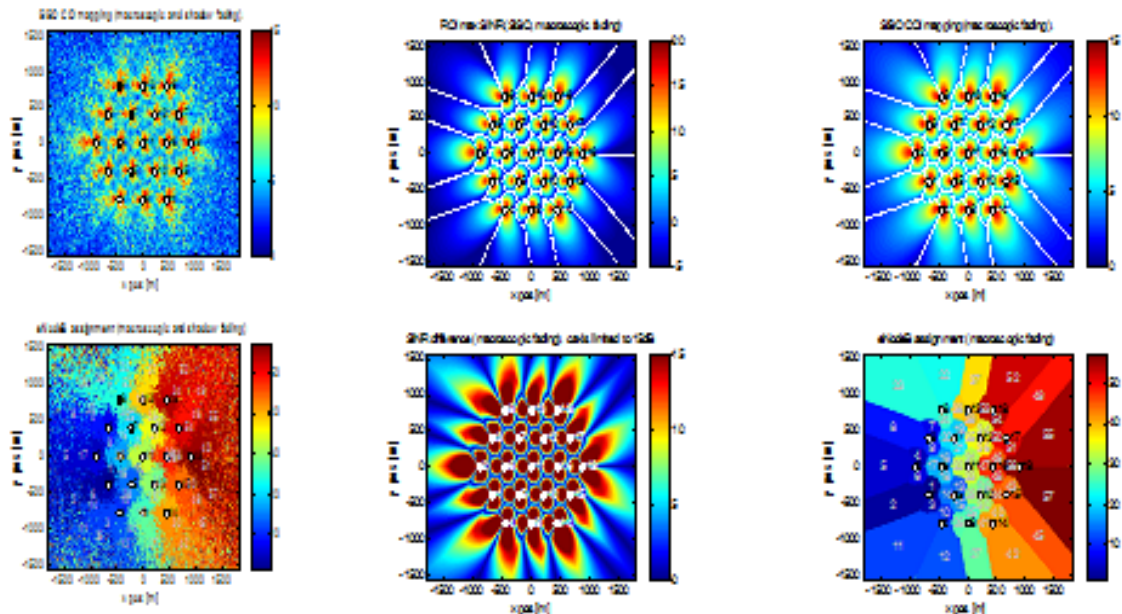

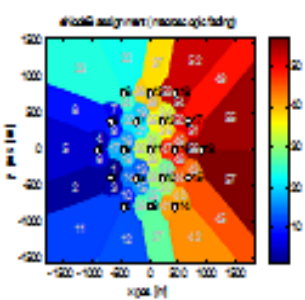

Fig.e: Macroscopic Fading

\section{B. Effect of UE distribution}

In this handout we describe the effects of mobility and radii under varied conditions. The UE distribution places a constant number of user equipment's per cell. The number of UE's per cell will be only one. It means each UE is having each cell. Moreover, the value of radial will be 2 which place around each 
eNodeB site a series of concentric rings with UEs. Next, a vector specifying the radius of each of the UE rings around the eNodeB sites which will be 1 and 10 . We choose the value of nUEs is 5 which are either a scalar specifying the number of UEs per ring or a vector specifying for each ring the number of UEs.

\section{UE distribution Radii $=1$}

Following are the results showing the effects of UE distribution for radii=1:

Below Fig.h shows the different plotting to each other. The fig.f clearly showing the CDF plot of UE average throughput up to $2.5 \mathrm{Mbps}$ which reached above $99.99 \%$ of UE's and also $60 \%$ of the nodes having UE average throughput up to $1 \mathrm{Mbps}$. Next, the fig.g showing the average spectral efficiency up to $2.5 \mathrm{bits} / \mathrm{cu}$ which reached for about $85 \%$ of UE's and also $79.99 \%$ of the nodes achieve the average UE spectral efficiency of $2 \mathrm{bits} / \mathrm{cu}$. Then the Fig. h shows the CDF plot of UE wideband SNR in which plotting on $\mathrm{x}$ axis starts from -15 to $20 \mathrm{~dB}$, the wideband SINR up to $-4 \mathrm{~dB}$ accomplished for about $1 \%$ of UE's and additional than $60 \%$ of the nodes achieve the UE wideband SINR of $1 \mathrm{~dB}$.

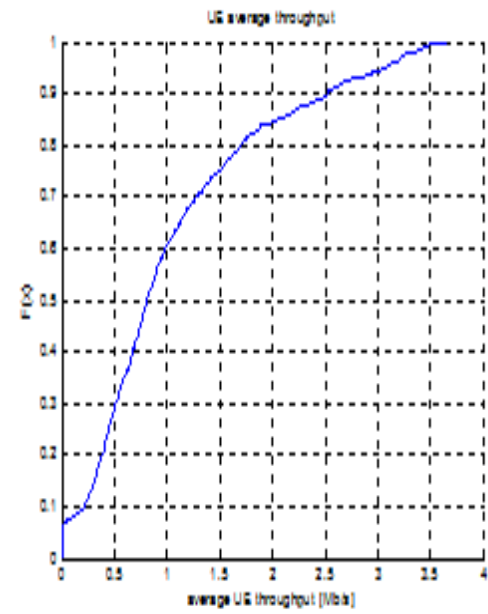

Fig.f: UE average throughput

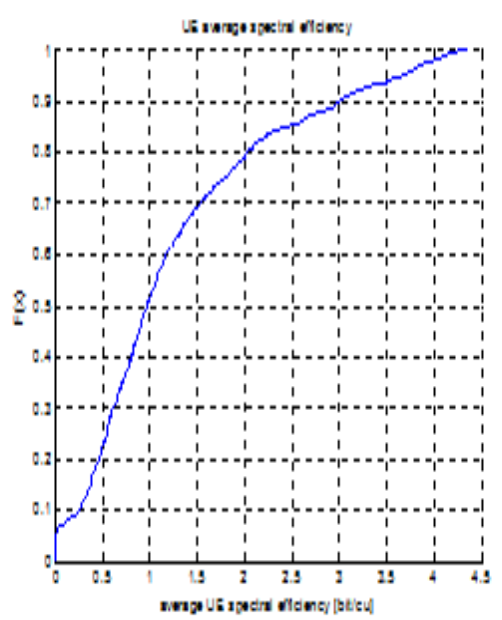

Fig.g: UE average spectral efficiency

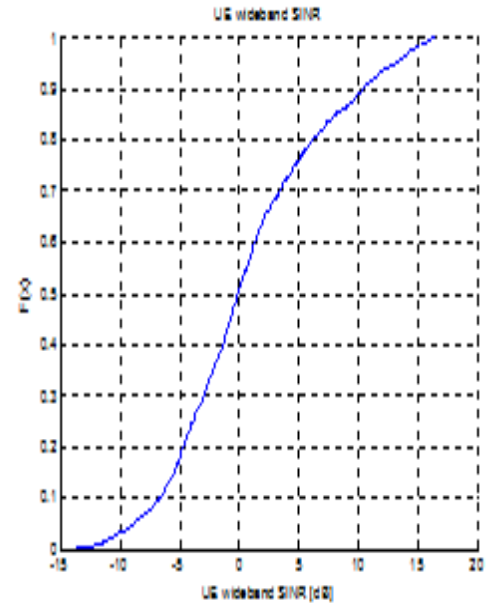

Fig.h: UE wideband SNR

UE distribution Radii $=10$

Below are the results for the effects of UE distribution for radii $=10$.

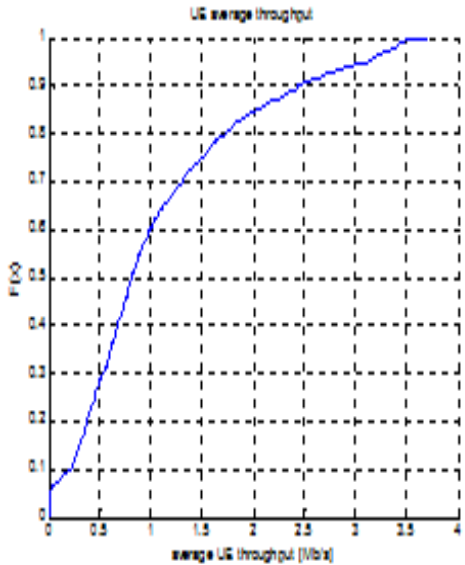

Fig.i: UE average throughput

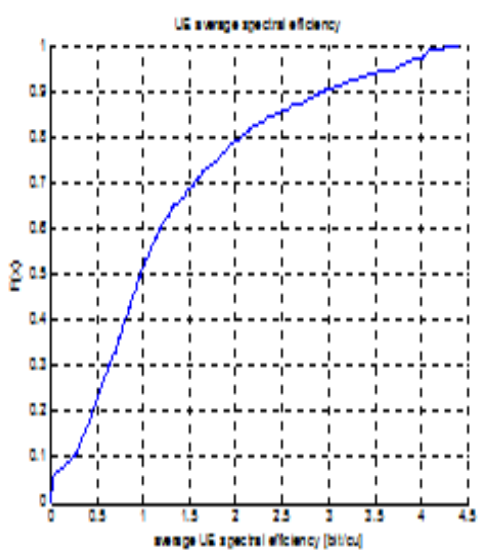

Fig.j: UE average spectral efficiency

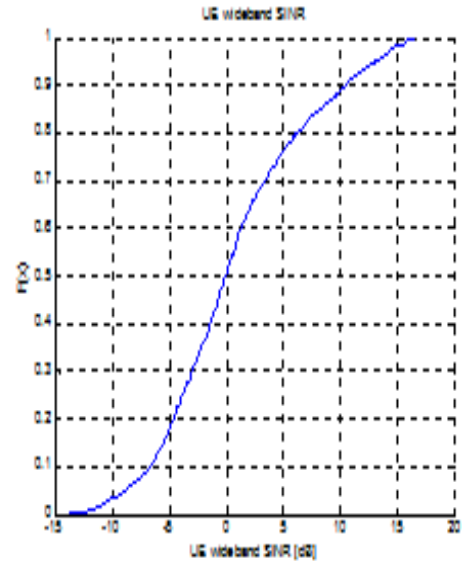

Fig.k: UE wideband SNR

Above Fig. i j,k shows the different plotting to each other. The fig.i clearly showing the CDF plot of UE average throughput up to 2 Mbps which reached above $85 \%$ of UE's and also $75 \%$ of the nodes having UE average throughput up to $1.5 \mathrm{Mbps}$. Next, the fig.j showing the average spectral efficiency up to $3 \mathrm{bits} / \mathrm{cu}$ which reached for about $91 \%$ of UE's and also $69 \%$ of the nodes achieve the average UE spectral efficiency of 1.5 bits/cu. Then the Fig. $\mathrm{k}$ shows the CDF plot of UE wideband SNR in which plotting on $\mathrm{x}$ axis starts from -15 to $20 \mathrm{~dB}$, the wideband SINR up to $0 \mathrm{~dB}$ accomplished for about $50 \%$ of UE's and additional than $77 \%$ of the nodes achieve the UE wideband SINR of $5 \mathrm{~dB}$. 


\begin{tabular}{|c|c|c|c|c|c|c|c|c|c|}
\hline $\mathrm{Sr}_{\mathrm{N}} \mathrm{N}_{0}$ & $\begin{array}{l}\text { UE_distrib } \\
\text { ution_radii }\end{array}$ & $\begin{array}{l}\text { Fairness } \\
\text { index }\end{array}$ & \begin{tabular}{|l} 
Peak \\
throughput \\
(Mbps) \\
\end{tabular} & $\begin{array}{l}\text { Avg. } \\
\text { throughpu } \\
\text { t(Mbps) } \\
\end{array}$ & $\begin{array}{l}\text { Edge UE } \\
\text { throughput(MI } \\
\text { bps) }\end{array}$ & $\begin{array}{l}\text { Avg. cell } \\
\text { throughput } \\
\text { (Mlops) } \\
\end{array}$ & $\begin{array}{l}\text { Avg.UE } \\
\text { throughput } \\
\text { (Mbps) } \\
\end{array}$ & $\begin{array}{l}\text { Avg.UE } \\
\text { spectral eff. } \\
\text { (bits } / \text { cu) } \\
\end{array}$ & $\begin{array}{l}\text { Rank } \\
\text { Indicators }\end{array}$ \\
\hline 1. & 1 & 0.603853 & 3.08 & 1.08 & 0.01 & 21.65 & 1.08 & 1.29 & $\begin{array}{l}\text { R1-92.58\% } \\
\text { R2.7.4.2\% }\end{array}$ \\
\hline 2. & 10 & 0.60421 & 3.12 & 1.08 & 0.02 & 21.61 & 1.08 & 1.29 & $\begin{array}{l}\text { R1-92.66\% } \\
\text { R2-7.7.34\% }\end{array}$ \\
\hline
\end{tabular}

Table 2: Effect of UE distribution

Above results showing about the effect of UE distribution. We have taken the values of UE distribution radii $=1$ and 10 . The speed of UE is $5 \mathrm{Km} / \mathrm{hr}$, nUEs of UE distribution $=5$. When the value of UE distribution radii is 1 then the fairness index is slightly smaller than that of radii=10. The peak throughput is smaller in the radii $=1$, but in radii $=10$, the fairness index is $3.12 \mathrm{Mbps}$. The average throughput, average UE throughput, average UE spectral efficiency remains same i.e. $1.08 \mathrm{Mbps}, 1.08 \mathrm{Mbps}, 1.29 \mathrm{bits} / \mathrm{cu}$. When the value of UE distribution is 15 then same results will takes place as that of the 10 . The average cell throughput will be greater in UE distribution radii 1 than that of UE distribution radii 10. There is slight change in rank indicators which clearly shows in table 2 between radii=1 and 10 .

\section{Effect of UE density}

In this section, there is a description of user's settings which includes UE receiver noise figure, UE thermal noise density, UE thermal noise density for SISO and MIMO LL-SL comparison. The speed of UE will be $8 \mathrm{Km} / \mathrm{hr}$ and distance between eNodeB is $450 \mathrm{~m}$. In this handout, mentioned values for UE per eNodeB will be 2, 25, scheduler= Round Robin, which is having additional configuration parameters for the scheduler i.e. fairness, alpha, beta. Following are the results showing for different values for UE per eNodeB's correspondingly UE reciever noise figure and UE thermal noise density.

\section{UE per eNodeB $=2$, Receiver noise figure $=13 \mathrm{~dB}$}

Below are the results for the effects of UE density by taking UE per eNodeB $=2$

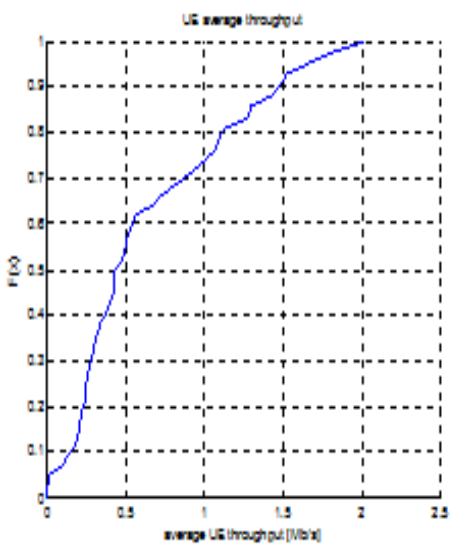

Fig.l: UE average throughput

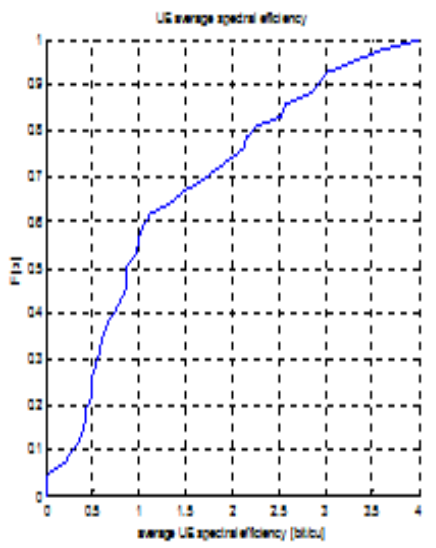

Fig.m: UE average spectral efficiency

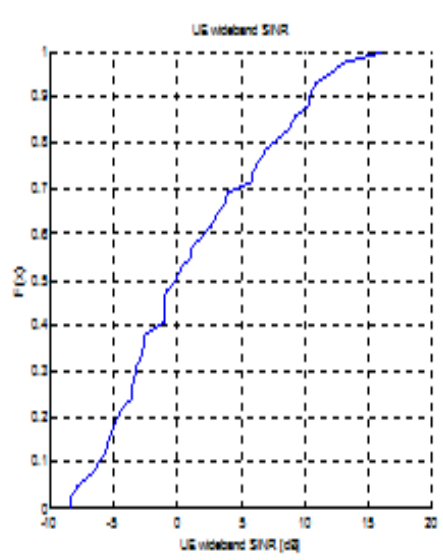

Fig.n: UE wideband SNR

Above Fig. 1,m,n shows the graphs which are different to each other. The fig.l clearly showing the CDF plot of UE average throughput up to $2 \mathrm{Mbps}$ which reached above $100 \%$ of UE's and also $60 \%$ of the nodes having UE average throughput up to $0.6 \mathrm{Mbps}$. Next, the fig.m showing the average spectral efficiency up to $1 \mathrm{bits} / \mathrm{cu}$ which reached for about 55\% of UE's and also $92 \%$ of the nodes achieve the average UE spectral efficiency of 3 bits/cu. Then the Fig. $n$ shows the CDF plot of UE wideband SNR in which plotting on $\mathrm{x}$ axis starts from -10 to $20 \mathrm{~dB}$, the wideband SINR up to $5 \mathrm{~dB}$ accomplished for about $70 \%$ of UE's and additional than $40 \%$ of the nodes achieve the UE wideband SINR of $-1 \mathrm{~dB}$. 

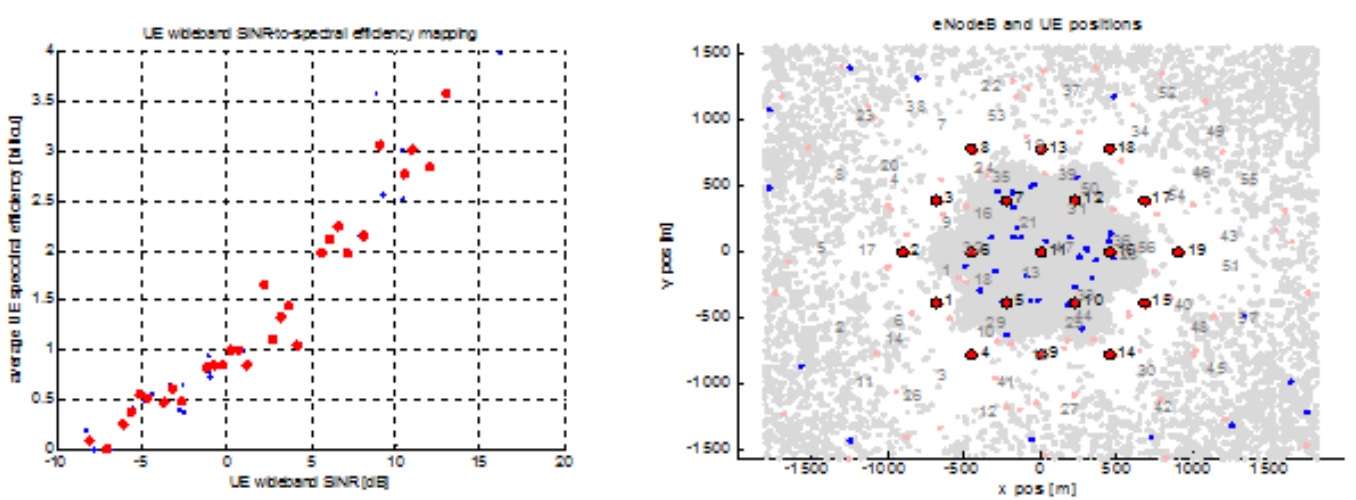

Fig.o: UE wideband SINR to spectral efficiency mapping

Fig.p: eNodeB and UE positions

\section{UE per eNodeB $=2$, Receiver noise figure $=20 \mathrm{~dB}$}

Below are the results for the effects of UE density by taking UE per eNodeB $=2$ and receiver noise figure $=20 \mathrm{~dB}$.

Below fig. q,r,s shows the graphs which are different to each other. The fig.q clearly showing the CDF plot of UE average throughput in which at $41 \%$ origin starts and up to $1.2 \mathrm{Mbps}$ which reached above $90 \%$ of UE's and also $81 \%$ of the nodes having UE average throughput up to $0.8 \mathrm{Mbps}$. Next, the fig.r showing the average spectral efficiency up to 1 bits/cu which reached for about $75 \%$ of UE's and also $99 \%$ of the nodes achieve the average UE spectral efficiency of $3.5 \mathrm{bits} / \mathrm{cu}$.

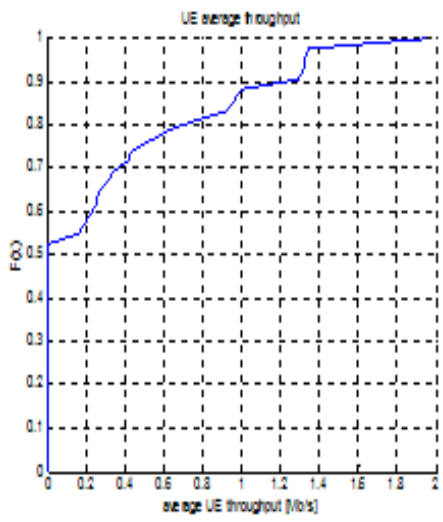

Fig.q: UE average throughput

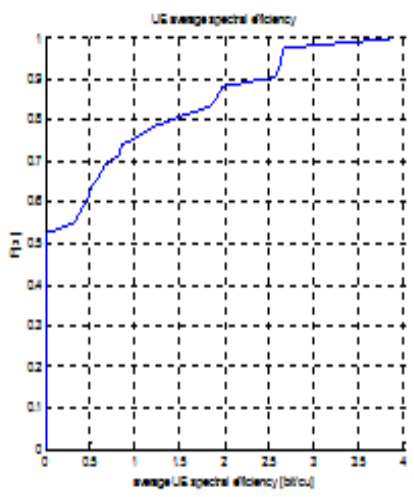

Fig.r: UE average spectral efficiency

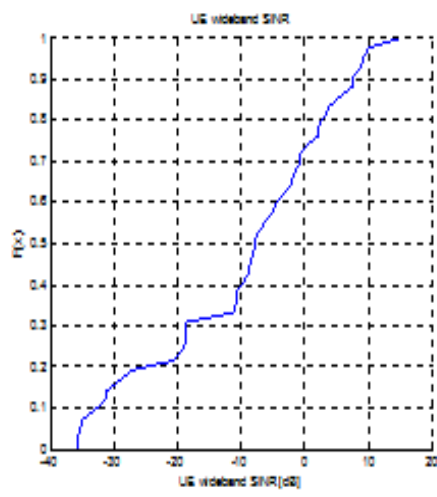

Fig.s: UE wideband SNR

Then the Fig. s shows the CDF plot of UE wideband SNR in which plotting on $\mathrm{x}$ axis starts from -40 to $20 \mathrm{~dB}$, the wideband SINR up to $-20 \mathrm{~dB}$ accomplished for about $22 \%$ of UE's and additional than $80 \%$ of the nodes achieve the UE wideband SINR of $3 \mathrm{~dB}$.

\begin{tabular}{|c|c|c|c|c|c|c|c|c|c|c|c|c|}
\hline \multicolumn{3}{|c|}{$\begin{array}{c}\text { Round Robin } \\
\text { Scheduler }\end{array}$} & \multicolumn{2}{|c|}{ Fairness $=0.5$} & \multicolumn{2}{|c|}{ Alpha $=1$} & \multicolumn{2}{|c|}{ Beta=1 } & \multicolumn{2}{|c|}{$\begin{array}{c}\text { Avg } \\
\text { RBs }=3.00 R B s \\
\end{array}$} & \multicolumn{2}{|c|}{ UE_per_eNodeB=2 } \\
\hline $\begin{array}{l}\text { Sr. } \\
\text { No. }\end{array}$ & $\begin{array}{c}\mathrm{UE} \\
\mathrm{RX} \\
\text { Noise } \\
\text { Figure } \\
\text { (dB) }\end{array}$ & $\begin{array}{c}\mathrm{UE} \\
\text { thermal } \\
\text { Noise } \\
\text { Density } \\
(\mathrm{SISO})(\mathrm{d} \\
\mathrm{Bm} H \mathrm{~Hz})\end{array}$ & $\begin{array}{c}\mathrm{UE} \\
\text { thermal } \\
\text { Noise } \\
\text { Density } \\
(\mathrm{MIN} N \mathrm{O})( \\
\mathrm{dBm} H \mathrm{~Hz}\end{array}$ & $\begin{array}{l}\text { UE thermal } \\
\text { Noise } \\
\text { Density(dB } \\
\mathrm{m} / \mathrm{Hz})\end{array}$ & \begin{tabular}{c|} 
Fairness \\
inder
\end{tabular} & 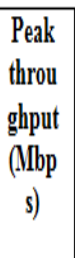 & $\begin{array}{l}\text { Arg. } \\
\text { throughpu } \\
\text { t(Mlops) }\end{array}$ & $\begin{array}{c}\text { Edge } \\
\text { UE } \\
\text { throug } \\
\text { hput } \\
\text { (Mllps) }\end{array}$ & $\begin{array}{c}\text { Avg. cell } \\
\text { throughput(III } \\
\text { bps) }\end{array}$ & $\begin{array}{l}\text { Arg. UE } \\
\text { throughp } \\
\text { ut(Mllpps) }\end{array}$ & $\begin{array}{l}\text { Arg. UE } \\
\text { spectral } \\
\text { eff.(bitscu) }\end{array}$ & Rank Indicators \\
\hline 1. & 13 & -120 & -100 & -160 & 0.61224 & 1.81 & 0.67 & 0.10 & 1.34 & 0.67 & 1.33 & $\begin{array}{l}\text { R1-95.48\% } \\
\text { R2-4.52\% }\end{array}$ \\
\hline 2. & 20 & -105 & -115 & -120 & 0.328956 & 1.32 & 0.35 & 0.00 & 0.70 & 0.35 & 0.70 & $\begin{array}{l}\text { R1-52.62\% } \\
\text { R2-47.38\% }\end{array}$ \\
\hline
\end{tabular}

Table 2: Simulation environment for UE-per_eNodeB=2 
Above are the results shows regarding the effects of UE distribution, In this we have select the round robin scheduler with additional parameters i.e. fairness $=0.5$, alpha $=1$, beta $=1$. The speed of UE is $8 \mathrm{Km} / \mathrm{hr}$, the transmitter power is $49 \mathrm{dBm}$, bandwidth $=1.4 * 10^{6} \mathrm{MHz}$. The model type is Gamma and UE distribution is constant UE per cell. We have taken the two values of receiver noise figure i.e. $13 \mathrm{~dB}, 20 \mathrm{~dB}$. When the value of Rx noise figure is $13 \mathrm{~dB}$, UE thermal noise density $(\mathrm{SISO})=-120 \mathrm{dBm} / \mathrm{Hz}$, UE thermal noise density $(\mathrm{MIMO})=$ $100 \mathrm{dBm} / \mathrm{Hz}$, UE thermal noise density $=-160 \mathrm{dBm} / \mathrm{Hz}$ then values of fairness index, average cell throughput, average throughput, average spectral efficiency is much higher than that of the UE receiver noise figure $20 \mathrm{~dB}$. The fluctuation in values takes place only because of taking the different values of UE receiver noise figure, UE thermal noise density (SISO), UE thermal noise density (MIMO), UE thermal noise density as well. The fairness will be good only when there is an increment in the value of noise figure.

\section{UE per eNodeB $=25$, Receiver noise figure $=13 \mathrm{~dB}$}

Below are the results for the effects of UE density by taking UE per eNodeB $=25$.

Following fig. t,u,v shows the graphs which are different to each other. The fig.t clearly showing the CDF plot of UE average throughput up to $0.1 \mathrm{Mbps}$ which reached above $82 \%$ of UE's and also $70 \%$ of the nodes having UE average throughput up to $0.06 \mathrm{Mbps}$.

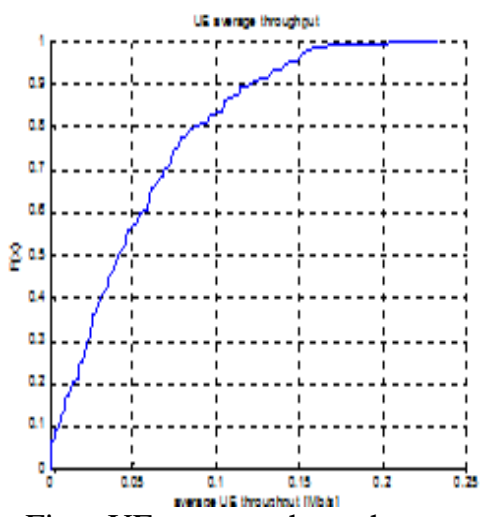

Fig.t: UE average throughput

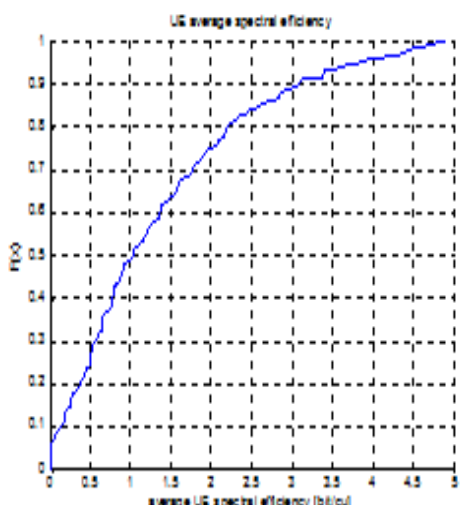

Fig.u: UE average spectral efficiency

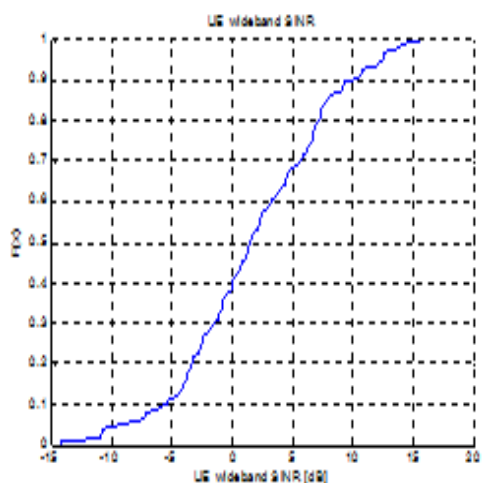

Fig.v: UE wideband SNR

Next, the fig.u showing the average spectral efficiency up to 3 bits/cu which reached for about $89 \%$ of UE's and also $98 \%$ of the nodes achieve the average UE spectral efficiency of $4.5 \mathrm{bits} / \mathrm{cu}$. Then the Fig. v shows the CDF plot of UE wideband SNR in which plotting on $\mathrm{x}$ axis starts from -15 to $20 \mathrm{~dB}$, the wideband SINR up to $-5 \mathrm{~dB}$ accomplished for about $11 \%$ of UE's and additional than $100 \%$ of the nodes achieve the UE wideband SINR of $15 \mathrm{~dB}$.

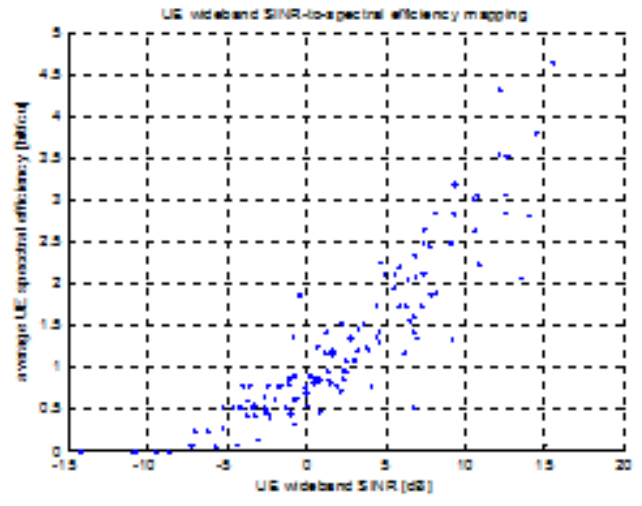

Fig.w: UE wideband SINR to spectral efficiency mapping

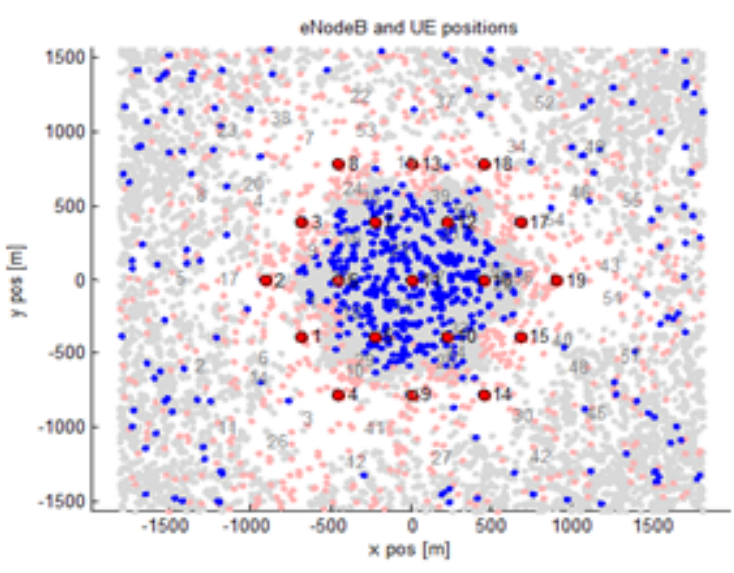

Fig.x: eNodeB and UE positions

UE per $\mathrm{eNode} B=25$, Receiver noise figure $=20 \mathrm{~dB}$

Following are the results for the effects of UE density by taking UE per eNodeB $=25$ and receiver noise figure $=20 \mathrm{~dB}$. 


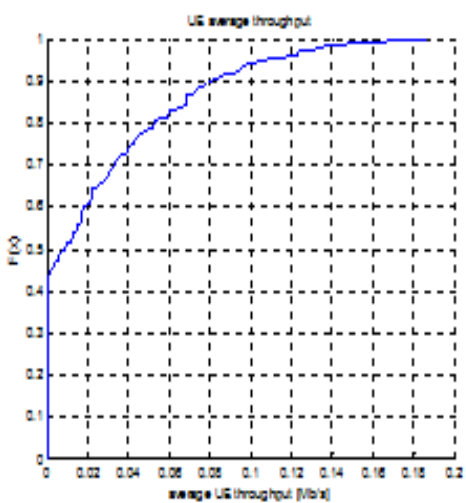

Fig.y: UE average throughput

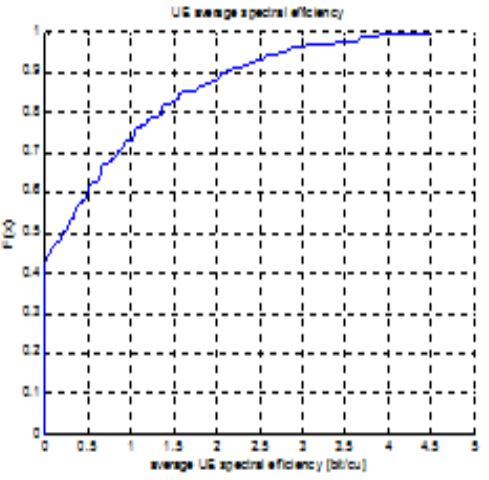

Fig.z: UE average spectral efficiency

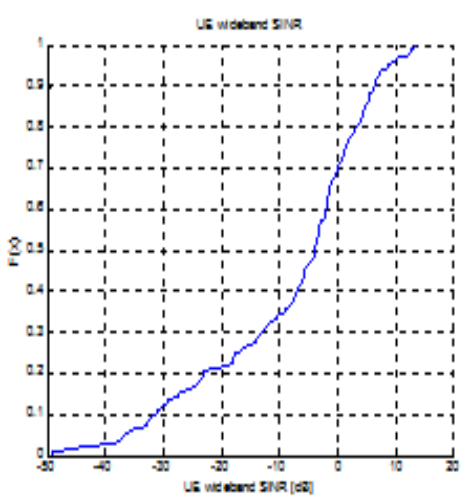

Fig.a1: UE wideband SNR

Above Fig. y,z,a1 shows the graphs which are dissimilar to each other. The fig.y clearly showing the CDF plot of UE average throughput and its origin starts above $40 \%$ and up to $0.08 \mathrm{Mbps}$ which reached above $90 \%$ of UE's and also $99 \%$ of the nodes having UE average throughput up to $0.14 \mathrm{Mbps}$. Next, the fig.z showing the average spectral efficiency up to 2 bits/cu which reached for about $88 \%$ of UE's and also $97 \%$ of the nodes achieve the average UE spectral efficiency of 3bits/cu. Then the Fig. al shows the CDF plot of UE wideband SNR in which plotting on $x$ axis starts from -50 to $20 \mathrm{~dB}$, the wideband SINR up to $-32 \mathrm{~dB}$ accomplished for about $10 \%$ of UE's and additional than $70 \%$ of the nodes achieve the UE wideband SINR of 0 $\mathrm{dB}$.

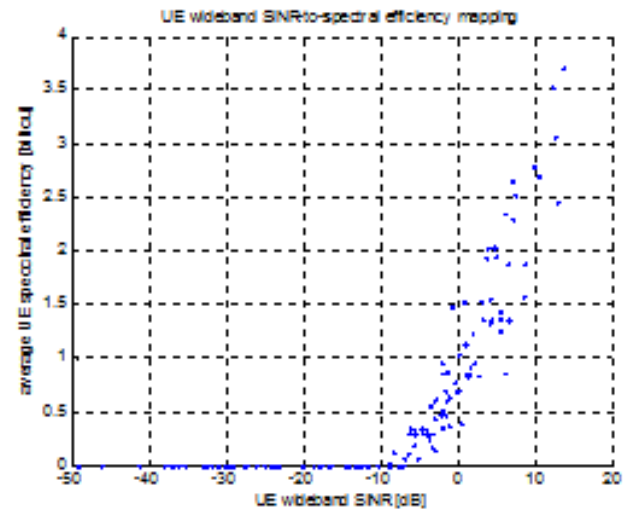

Fig.a2: UE wideband SINR to spectral efficiency mapping

\begin{tabular}{|c|c|c|c|c|c|}
\hline $\begin{array}{c}\text { Round Robin } \\
\text { Scheduler }\end{array}$ & Fairness=0.5 & Alpha=1 & Beta=1 & $\begin{array}{c}\text { Avg } \\
\text { RBs=0.24RBs }\end{array}$ & UE_per_eNodeB=25 \\
\hline
\end{tabular}

\begin{tabular}{|c|c|c|c|c|c|c|c|c|c|c|c|}
\hline $\begin{array}{l}\text { Sr. } \\
\text { No. }\end{array}$ & $\begin{array}{c}\text { UE } \\
\text { RX } \\
\text { Noise } \\
\text { Figur } \\
\text { e (dB) }\end{array}$ & $\begin{array}{l}\text { UE thermal } \\
\text { Noise } \\
\text { Density } \\
\text { (SISO) }(\mathrm{dBm} / \\
\mathrm{Hz})\end{array}$ & $\begin{array}{c}\mathrm{UE} \\
\text { thermal } \\
\text { Noise } \\
\text { Density } \\
(\mathrm{MIN})( \\
\mathrm{dBm} / \mathrm{Hz})\end{array}$ & $\begin{array}{l}\text { UE thermal } \\
\text { Noise } \\
\text { Density(dBm } \\
\text { Hz })\end{array}$ & $\begin{array}{l}\text { Fairness } \\
\text { index }\end{array}$ & $\begin{array}{c}\text { Peak } \\
\text { throughput(M } \\
\text { bps) }\end{array}$ & $\begin{array}{c}\text { Avg. } \\
\text { throughput } \\
\text { (Mbps) }\end{array}$ & $\begin{array}{c}\text { Avg. cell } \\
\text { throughput((M } \\
\text { bps) }\end{array}$ & $\begin{array}{c}\text { Avg. UE } \\
\text { throughput( } \\
\text { Mbps) }\end{array}$ & $\begin{array}{c}\text { Avg. } \\
\text { UE } \\
\text { spectral } \\
\text { eff.(bits/ } \\
\text { cu) }\end{array}$ & $\begin{array}{c}\text { Rank } \\
\text { Indicators }\end{array}$ \\
\hline 1. & 13 & -120 & -100 & -160 & 0.593582 & 0.14 & 0.05 & 1.34 & 0.05 & 1.36 & $\begin{array}{l}\text { R1-98.42\% } \\
\text { R2-1.58\% }\end{array}$ \\
\hline 2. & 20 & -105 & -115 & -120 & 0.3417 & 0.11 & 0.03 & 0.67 & 0.03 & 0.69 & $\begin{array}{l}\text { R1-90.53\% } \\
\text { R2-9.47\% }\end{array}$ \\
\hline
\end{tabular}

Table 3: Simulation environment for UE-per_eNodeB=25

Above are the results shows regarding the effects of UE distribution, In this we have taken the two values of receiver noise figure i.e. $13 \mathrm{~dB}, 20 \mathrm{~dB}$. When the value of Rx noise figure is $13 \mathrm{~dB}$, UE thermal noise density $(\mathrm{SISO})=-120 \mathrm{dBm} / \mathrm{Hz}$, UE thermal noise density $(\mathrm{MIMO})=-100 \mathrm{dBm} / \mathrm{Hz}$, UE thermal noise density= - 
$160 \mathrm{dBm} / \mathrm{Hz}$ then values of fairness index, average cell throughput, average throughput, average spectral efficiency is much higher than that of the UE receiver noise figure $=20 \mathrm{~dB}$. The fluctuation in values takes place since of taking the dissimilar values of UE receiver noise figure, UE thermal noise density (SISO), UE thermal noise density (MIMO), UE thermal noise density as well. The fairness will be good only when there is an augmentation in the value of noise figure. The above table clearly displays that the fairness index is lower when the value of receiver noise figure $=20 \mathrm{~dB}$.

\section{Conclusions}

In this sequence we have perceived the effect of speed, effect of UE distribution and effect of UE density. We have scrutinized that by taking different values of mobility then the flower diagrams, shadow fading, and macroscopic fading takes place. Moreover there are lots of effects of UE distribution takes place when there is change in a value of radii of UE distribution. In this handout we have taken the values of UE radii i.e. 1 and 10. The fairness will increase only when the radii value will be more. Moreover we have also describe the effects of UE distribution in which we consider the values of eNodeB's, receiver noise figure, thermal noise density as well. We conclude that if the value of eNodeB's decreases then fairness increases. Because lesser no. of people does not use same channel so that fairness will ultimately increases. The main objective of this paper is to display the effects of mobility, effects of density, effects of distribution on many users. This suggestion permits individuals who are concerned in wireless proclamation gets connected with the wireless association concerning the evolution of LTE.

\section{Acknowledgment}

The author wishes to sanction Dr. Jyoteesh Malhotra and GNDU for keeping them judicious and compassionate for this work.

\section{Reference}

[1] M.T.K., A.R.H, H.M. A. B. Farid, Adil M. J. S., Ibrahim K.R., Performance Comparison between Round Robin and Proportional Fair Scheduling Methods for LTE, Department of the Electrical and Electronic Engineering Islamic University of Technology Dhaka, Bangladesh.

[2] Habaebi, et al., "Comparison Between Scheduling Techniques in Long Term Evolution”, IIUM Engineering Journal, Vol. 14, no. 1, pp. 66-75, 2013.

[3] S. Schwarz, C. Mehlfuhrer and M. Rupp, "Low Complexity Approximate Maximum Throughput Scheduling for LTE", 44th Annual Asilomar conference on Signals, Systems and Computers, California, 2010.

[4] Ericsson, "LTE-an introduction", White Paper, June 2009.

[5] "Fair downlink scheduling algorithm for 3gpp LTE networks", mecs, Vol. 6, pp- 34-41, 2013.

[6] M. H. Habaebi, J. Chebil, A.G. Al-Sakkaf and T. H. Dahawi, "Comparison between Scheduling techniques in long term evolution", IIUM Engineering Journal, Vol. 14, no. 1, pp 66-75, 2013.

[7] E.Dahlman, S.Parkvall, J.Skold, P.Beming, "3G Evolution HSPA and LTE for Mobile Broadband", Elsevier, 2008. 